\title{
Quantum oscillations probe the Fermi surface topology of the nodal-line semimetal CaAgAs
}

\author{
Y. H. Kwan $\odot,{ }^{1, *}$ P. Reiss $\odot,{ }^{2}$ Y. Han, ${ }^{2}$ M. Bristow $\odot,{ }^{2}$ D. Prabhakaran, ${ }^{2}$ D. Graf,${ }^{3}$ A. McCollam, ${ }^{4}$ \\ S. A. Parameswaran ${ }^{\circ},{ }^{1}$ and A. I. Coldea $\oplus^{2, *}$ \\ ${ }^{1}$ The Rudolf Peierls Centre for Theoretical Physics, Department of Physics, University of Oxford, Oxford OX1 3NP, United Kingdom \\ ${ }^{2}$ Clarendon Laboratory, Department of Physics, University of Oxford, Parks Road, Oxford OX1 3PU, United Kingdom \\ ${ }^{3}$ National High Magnetic Field Laboratory, Florida State University, Tallahassee, Florida 32310, USA \\ ${ }^{4}$ High Field Magnet Laboratory (HFML-EMFL), Radboud University, 6525 ED Nijmegen, The Netherlands
}

(Received 31 July 2019; accepted 23 December 2019; published 6 March 2020)

\begin{abstract}
Nodal semimetals are a unique platform to explore topological signatures of the unusual band structure that can manifest by accumulating a nontrivial phase in quantum oscillations. Here we report a study of the de Haas-van Alphen oscillations of the candidate topological nodal line semimetal CaAgAs using torque measurements in magnetic fields up to $45 \mathrm{~T}$. Our results are compared with calculations for a toroidal Fermi surface originating from the nodal ring. We find evidence of a nontrivial $\pi$ phase shift only in one of the oscillatory frequencies. We interpret this as a Berry phase arising from the semiclassical electronic Landau orbit which links with the nodal ring when the magnetic field lies in the mirror $(a b)$ plane. Furthermore, additional Berry phase accumulates while rotating the magnetic field for the second orbit in the same orientation which does not link with the nodal ring. These effects are expected in CaAgAs due to the lack of inversion symmetry. Our study experimentally demonstrates that $\mathrm{CaAgAs}$ is an ideal platform for exploring the physics of nodal line semimetals and our approach can be extended to other materials in which trivial and nontrivial oscillations are present.
\end{abstract}

DOI: 10.1103/PhysRevResearch.2.012055

Introduction. The theoretical and experimental study of topological materials has been one of the most active fields in condensed matter physics over the last decade. Topological nodal-line semimetals (TNLSMs) [1-4] are recent additions to the roster of topologically nontrivial systems. The band structure of these materials is characterized by the degeneracy of conduction and valence bands along a one-dimensional line in the three-dimensional (3D) Brillouin zone. Unlike the point degeneracies in Weyl semimetals that are robust to generic perturbations, the stability of nodal lines requires additional symmetries, such as inversion and time-reversal (as in $\mathrm{Cu}_{3} \mathrm{PdN}$ [5,6]), reflection (e.g., $\mathrm{PbTaSe}_{2}$ [7]), or nonsymmorphic symmetries (as in $\mathrm{ZrSiS}$ [8,9]). TNLSMs can host nearly flat drumhead surface states [10,11], and are predicted to exhibit an array of unconventional electromagnetic properties [12] and correlation effects [13]. Although in principle the stability of TNLSMs with spin-orbit coupling (SOC) against forming topological insulators/semimetals is a matter of detail that depends on the protecting symmetries, in practice the nodal-line description remains a good approximation for small SOC.

Recent first-principles calculations and theoretical analysis suggest that the noncentrosymmetric pnictides $\mathrm{CaAgX}$

\footnotetext{
${ }^{*}$ Corresponding authors: yves.kwan@physics.ox.ac.uk; amalia.coldea@physics.ox.ac.uk

Published by the American Physical Society under the terms of the Creative Commons Attribution 4.0 International license. Further distribution of this work must maintain attribution to the author(s) and the published article's title, journal citation, and DOI.
}

( $X=$ As, $\mathrm{P}$ ) are potential TNLSMs [14]. An unusual feature of these compounds is that at the intrinsic Fermi energy, no other structures apart from the nodal ring are predicted to be present. This allows experiments to directly examine properties of the nodal line without contamination from trivial Fermi pockets, in contrast to other proposed candidates. Hence, $\mathrm{CaAg} X$ has been dubbed the "hydrogen atom" of TNLSM phenomenology $[15,16]$. Transport studies indicate that the Fermi energy of CaAgAs is sufficiently close to the nodal line that the dispersion is within the linear regime, whereas its phosphide counterpart exhibits significant band curvature [17]. Therefore, experimental effort so far [angle-resolved photoemission spectroscopy (ARPES) [15,18,19] and transport [16-18]] has focused on $\mathrm{CaAgAs}$, which has a toroidal Fermi surface due to intrinsic hole doping caused by Ag deficiencies [16].

In this Rapid Communication, we report a quantum oscillation study of single crystals of $\mathrm{CaAgAs}$ observed via torque measurements in magnetic fields up to $45 \mathrm{~T}$. By mapping the orientation dependence of de Haas-van Alphen oscillations, we provide evidence of a small toroidal Fermi surface with light cyclotron effective masses. We use a circular torus model and band structure calculations to discuss the origin of different extremal orbits and their expected oscillations. Building on this, we perform a phase analysis and find evidence of a nontrivial Berry phase arising from semiclassical electron orbits which link with the nodal ring. Our results provide experimental and computational evidence of nontrivial quantum oscillations spectra in an ideal TNLSM candidate.

Fermi surface topology of CaAgAs. CaAgAs crystallizes in the hexagonal space group $P \overline{6} 2 m$, and the corresponding point symmetry group is $D_{3 \mathrm{~h}}$ [20], which contains a mirror plane perpendicular to the $c$ axis but no inversion. Without SOC, 

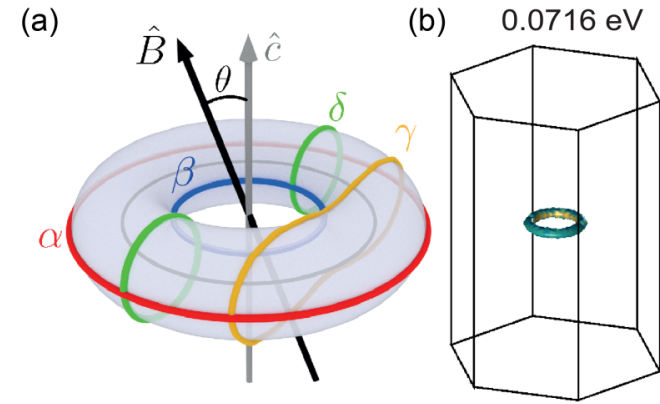

(c)

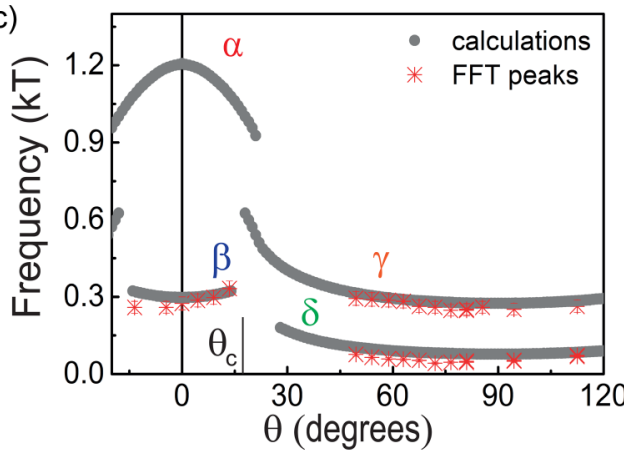

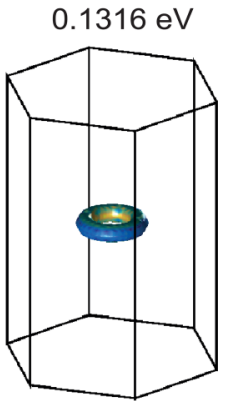

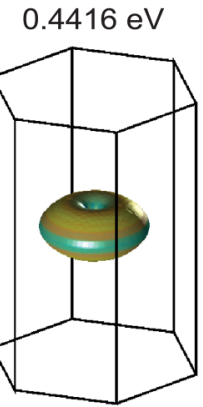

$0.5716 \mathrm{eV}$

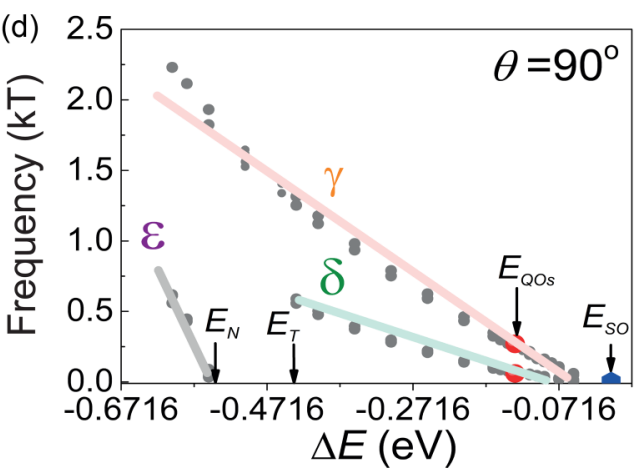

FIG. 1. Fermi surface topology of CaAgAs. (a) Torus Fermi surface showing the relative orientation $(\theta)$ of the magnetic field axis $\hat{B}$ with respect to the crystalline $c$ axis. $\alpha$ and $\beta$ are the semiclassical extremal orbits for $\hat{B} \| \hat{c}\left(\theta=0^{\circ}\right)$, and $\delta$ and $\gamma$ for $\hat{B} \perp \hat{c}\left(\theta=90^{\circ}\right)$. Only $\delta$ links with the nodal ring and is expected to have a nontrivial Berry phase. (b) Band structure calculation of the Fermi surface of CaAgAs for different values of hole doping below the theoretical Fermi energy, plotted in the first Brillouin zone. (c) Band structure calculations for the quantum oscillation frequencies as a function of $\theta$ with $E_{F}$ shifted by $\sim 0.13 \mathrm{eV}$ due to hole doping. Red asterisks are experimental data points. (d) Predicted quantum oscillation frequencies as a function of hole doping for $\theta=90^{\circ}$. At perfect stoichiometry $E=E_{\mathrm{SO}}$ there is no FS. $E_{\mathrm{QO}}$ is the Fermi energy based on experimental quantum oscillations. The torus closes at $E_{T}$, and a new band occurs inside at $E_{N}$.

the mirror symmetry pins the nodal ring to the $k_{z}=0$ plane and protects it from gapping out since the crossing bands have opposite mirror eigenvalues. In the presence of SOC, an energy gap of $\sim 0.07 \mathrm{eV}$ opens and CaAgAs becomes a strong 3D topological insulator [14]. However, if the doping is greater than the SOC gap, many of the features of the underlying nodal-ring physics, such as the Fermi surface topology, remain relevant.

Quantum oscillations in TNLSMs have been predicted to exhibit a peculiar behavior [21-23] and can accumulate a topological Berry phase. A qualitative understanding of the quantum oscillations of CaAgAs can be gained by considering the limit of a circular torus Fermi surface (FS) [Fig. 1(a)], which is expected to capture the bulk behavior of the nodal ring at small doping. The fundamental frequency $F=\frac{\hbar}{2 \pi e} A_{k}$ of quantum oscillations is determined by orbits on the FS that enclose locally extremal momentum-space area $A_{k}$ [24]. Complex Fermi surfaces can have multiple extremal orbits, and the experimental response will be a superposition of these oscillations.

Band structure calculations show the evolution of the Fermi surface with hole doping in Fig. 1(b). The nodal ring of $\mathrm{CaAgAs}$ is centered at the Brillouin zone center, $\Gamma$, and disperses linearly $[15,18,19]$. The toroidal FS increases in size with hole doping, and eventually closes at an energy $E_{T}$. Further hole doping above $0.5 \mathrm{eV}$ leads to the appearance of an additional band at $E_{N}$ inside the large Fermi pocket.

We find that both band structure calculations and the torus model [see Supplemental Material (SM) for details [25]] predict two distinct oscillation frequencies for all magnetic field directions, as shown in Fig. 1(c) (see also Figs. S3 and $\mathrm{S} 5$ in the SM [25]). There is a critical angle, $\theta_{c}$, that separates two different angular regimes. Firstly, at low angles $\left(\theta<\theta_{c}\right)$ close to $\hat{B} \| \hat{c}\left(\theta=0^{\circ}\right)$, the extremal orbits are $\alpha$ and $\beta$ shown in Fig. 1(a). Neither of them link with the nodal ring. With increasing angle $\theta$, the larger orbit $\alpha$ monotonically decreases in frequency, while the smaller orbit $\beta$ increases monotonically [Fig. 1(c)]. Secondly, for high angles $\left(\theta_{c}<\theta \leqslant 90^{\circ}\right)$, the handle orbit $\delta$ of the torus is topological since it links with the nodal ring [Fig. 1(a)]. There is also an orbit $\gamma$ located away from the center of the torus, which does not link with the nodal ring. Both $\delta$ and $\gamma$ monotonically increase in frequency as $\theta$ is reduced from $90^{\circ}$ [Fig. 1(c)]. In the vicinity of $\theta_{c}$, we expect magnetic breakdown [24] because different orbits approach each other closely in $k$ space. This is expected to manifest itself in breakdown orbits with a quasirandom spectrum and a suppression of the oscillation amplitude [26].

Experimental details. Single crystals of CaAgAs were grown by the flux method [17] and form hexagonal rods along the $c$ axis. Torque measurements were performed on a small single crystal of $<100 \mu \mathrm{m}$ at National High Magnetic Field Laboratory in Tallahassee up to $45 \mathrm{~T}$ and High Magnetic Field Laboratory in Nijmegen up to $38 \mathrm{~T}$ using a piezocantilever, a single-axis rotator (error less than $3^{\circ}$ ), and a variable-temperature cryostat. This crystal was verified by x-ray studies to be a single-phase crystal (Fig. S7). Band structure calculations were performed using WIEN $2 \mathrm{~K}$ with SOC and generalized gradient approximation on a 

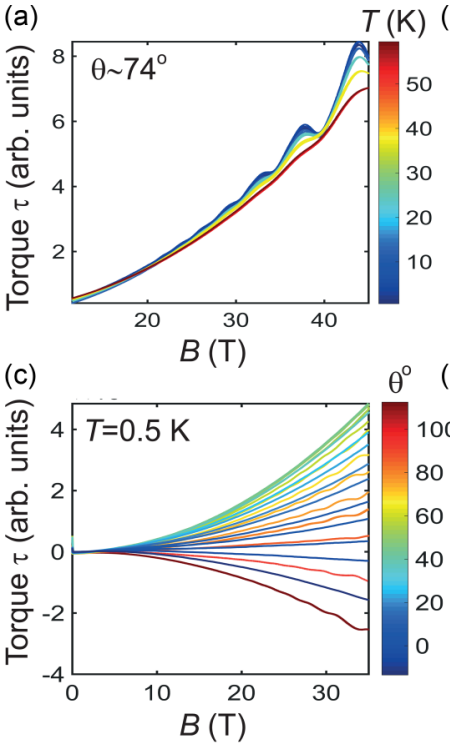

$\theta^{\circ}(\mathrm{d})$

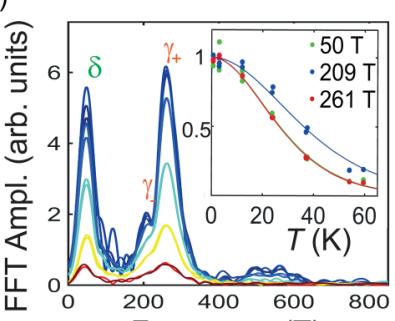

Frequency $(\mathrm{T})$

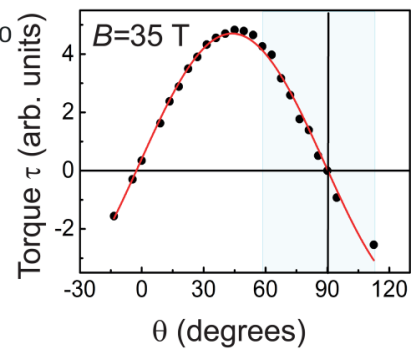

(e)

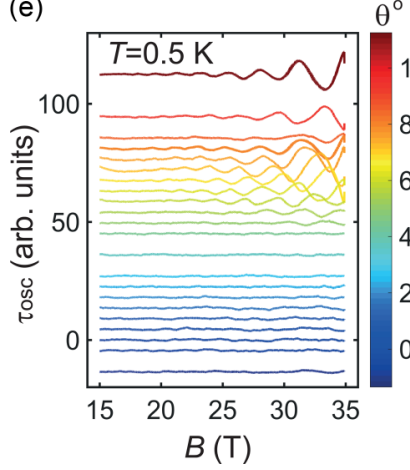

(f)

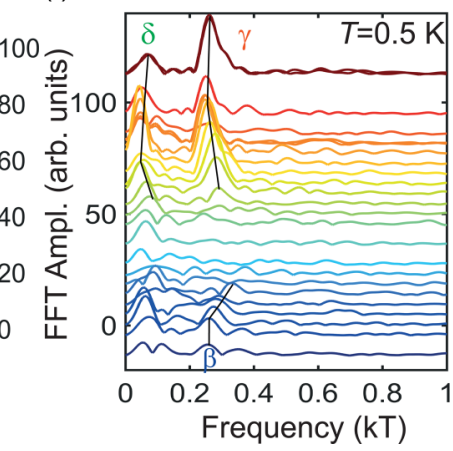

FIG. 2. Quantum oscillation experiments in CaAgAs. (a) Total torque for a fixed field orientation $\left(\theta \sim 74^{\circ}\right)$ at different constant temperatures. (b) Fast Fourier transform frequency spectrum of (a) after subtraction of a polynomial background. Inset shows the temperature dependence of different FFT amplitudes fitted to the damping factor $R_{T}$ to extract the effective masses. (c) Torque data for different orientations in magnetic field at $0.5 \mathrm{~K}$. (d) Overall amplitude of torque in $35 \mathrm{~T}$ fitted by a $\sin 2 \theta$ dependence indicates the symmetry axes where torque vanishes. (e) Oscillatory torque, $\tau_{\text {osc }}(B)$, obtained by subtracting a background from (c). (f) Waterfall plot showing the evolution of the frequency spectrum as a function of $\theta$. The angle is indicated by the vertical axis and the color bar in (e).

mesh containing $30 \times 30 \times 44 k$ points. Lattice parameters are $a=b=7.2040(6) \AA$ and $c=4.2700$ (4) $\AA$.

Quantum oscillation experiments in CaAgAs. Figure 2 shows evidence of quantum oscillations in $\mathrm{CaAgAs}$ from torque measurements as a function of magnetic field for different orientations. In order to isolate the oscillatory signal, a quadratic polynomial is subtracted from the raw data, since the total torque is given by $\boldsymbol{\tau}=\mathbf{M} \times \mathbf{B}$. The amplitude of quantum oscillations can be described by the standard Lifshitz-Kosevich (LK) formula [24], as detailed in the SM [25]. The oscillatory part of torque is given by the sum of the contributions from all extremal orbits on the Fermi surface for a particular orientation in magnetic field: $\tau_{\mathrm{osc}}(B) \sim$ $\sum_{i} A_{i} B^{3 / 2} R_{T} R_{D} R_{S} \sin \left(2 \pi F_{i} / B+\phi_{i}\right)$, where $R_{T}$ accounts for finite temperature effects, $R_{D}$ for impurity scattering, and $R_{S}$ for spin-splitting effects.

Figure 2(a) shows torque measurements up to $45 \mathrm{~T}$ measured for different constant temperatures up to $60 \mathrm{~K}$. The fast Fourier transform (FFT) spectra indicate several frequencies with a dominant frequency close to $260 \mathrm{~T}$, a small shoulder around $210 \mathrm{~T}$, and a low-frequency peak close to $50 \mathrm{~T}$, as shown in Fig. 2(b). By fitting the $R_{T}$ damping term to the temperature dependence of the quantum oscillation amplitude, we extract very light masses of $0.12(1) m_{e}$ for the 50 and $260 \mathrm{~T}$ frequencies and 0.092(5) $m_{e}$ for the $210 \mathrm{~T}$ frequency, as shown in the inset of Fig. 2(b). Similarly, fitting the $R_{D}$ term leads to a Dingle temperature of $65 \mathrm{~K}$, a scattering time of $0.019 \mathrm{ps}$, and a mean free path of $420 \AA$ [see Fig. S2(c)]. The proximity of the two largest frequencies may indicate that they arise from Zeeman splitting of a single orbit. This scenario leads to a $g$ factor of $g \simeq 7$ for $\theta=74^{\circ}$ (see SM [25] for additional discussion on spin-splitting effects [27]).

In order to understand the origin of the observed frequencies, we performed a series of measurements at various orientations $\theta$, as shown in Fig. 2(c) [see also Fig. S7(a)]. The angle-dependent Fourier spectra are summarized in Fig. 2(f). When the magnetic field is nearly perpendicular to the $c$ axis, there are two distinct oscillations at 50 and $260 \mathrm{~T}$, which we identify as $\delta$ and $\gamma$, respectively. The position of $\theta=90^{\circ}$ $(\hat{B} \perp \hat{c})$ was deduced based on the suppression of the torque at this high-symmetry orientation, as shown in Fig. 2(d). As $\hat{B}$ rotates from the $a-b$ plane to the $c$ axis, the $\delta$ and $\gamma$ oscillations increase in frequency as expected from band structure calculations in Fig. 1(c). The oscillations persist down to $\theta=50^{\circ}$, suggesting that the critical angle $\theta_{c}$ is below this value. At low angles close to $B \| c$, we also observe an FFT peak around $260 \mathrm{~T}$ which is consistent with the $\beta$ orbit.

Comparing the experimental frequencies with those predicted by band structure calculations shown in Fig. 1(d) (additional simulations in Figs. S3, S4, and S5 in the SM [25]), we estimate that our system is hole doped with an energy shift of $\Delta E \simeq 0.1316 \mathrm{eV}$. This doping level generates frequencies of $67.5 \mathrm{~T}$ for $\delta$ and $245 \mathrm{~T}$ for $\gamma$, in good agreement with the experimental data in Fig. 2. This energy shift predicts a critical angle $\theta_{c} \simeq 21^{\circ}$ and frequencies of $\sim 1200 \mathrm{~T}$ for $\alpha$ and $\sim 300 \mathrm{~T}$ for $\beta$, as shown in Fig. S3. Based on these parameters, we estimate the carrier density to be $5.2 \times 10^{19} \mathrm{~cm}^{-3}$, close to the experimental value $7.5 \times 10^{19} \mathrm{~cm}^{-3}$ obtained via transport measurements [17]. ARPES measurements on CaAgAs have reported a wide range of hole-doping levels with energy shifts from $0.05 \mathrm{eV}$ [19] to $0.5 \mathrm{eV}[15,18]$.

Phase analysis of quantum oscillations. The Berry phase, $\phi_{B}$, is a signature of nontrivial band topology, and in TNLSMs with combined inversion and time-reversal symmetry $\hat{\mathcal{T}} \hat{\mathcal{I}}$, the Berry phase along any closed contour in momentum space is either 0 or $\pi$. Only contours that link nontrivially with the nodal ring pick up a $\pi$ Berry phase - this is precisely the $\mathbb{Z}_{2}$ invariant describing TNSLMs protected by $\hat{\mathcal{T}} \hat{\mathcal{I}}$. On the other hand, $\mathrm{CaAgAs}$ is a noncentrosymmetric compound whose nodal ring (in the absence of SOC) is protected instead by mirror symmetry, giving rise to a $\mathbb{Z}$ mirror invariant. However, the Berry phases remain quantized but only for those orbits defined at high-symmetry directions, as shown in Table I and derived in the SM [25]. 
TABLE I. The various phases for the four types of extremal orbits of $\mathrm{CaAgAs}$. The phase values listed in the last two columns are strictly valid only for the magnetic field orientation shown in brackets.

\begin{tabular}{lcrcc}
\hline \hline Orbit & $\operatorname{sgn}\left(\frac{d F}{d \theta}\right)$ & \multicolumn{1}{c}{$\phi_{3 \mathrm{D}}$} & $\phi_{B}$ & $\phi_{i}$ \\
\hline$\alpha\left(\theta=0^{\circ}\right)$ & - & $\pi / 4$ & 0 & $5 \pi / 4$ \\
$\beta\left(\theta=0^{\circ}\right)$ & + & $-\pi / 4$ & 0 & $3 \pi / 4$ \\
$\gamma\left(\theta=90^{\circ}\right)$ & - & $\pi / 4$ & 0 & $5 \pi / 4$ \\
$\delta\left(\theta=90^{\circ}\right)$ & - & $-\pi / 4$ & $\pi$ & $-\pi / 4$ \\
\hline \hline
\end{tabular}

The phase factor of quantum oscillations for each orbit $\phi_{i}=-\pi+\phi_{B}+\phi_{3 \mathrm{D}}$ depends on $\phi_{B}$. For hole carriers, $\phi_{3 \mathrm{D}}= \pm \pi / 4$ depends on whether the extremal orbit has locally minimum or maximum area, respectively [24]. The phase shifts, in the absence SOC, can be predicted for each (a)
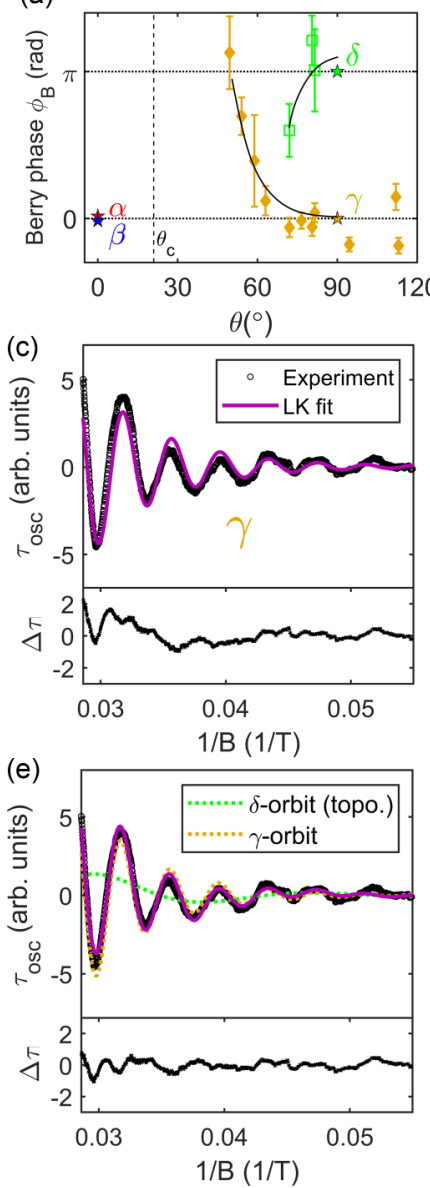

(b)
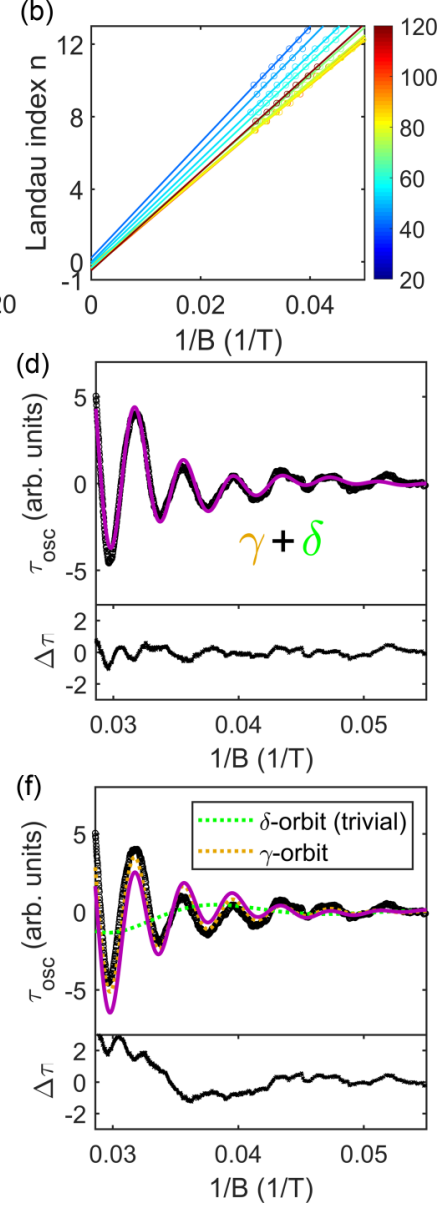

FIG. 3. Phase analysis of quantum oscillations. (a) Berry phases extracted using a two-component LK formula. The stars represent theoretical predictions in the absence of SOC. (b) Landau fan diagram for $\gamma$ [see Fig. S6(c)]. (c) One-component LK fit to $\gamma$ for a field sweep at $\theta=81^{\circ}$. Lower panel shows residuals. (d) Twocomponent LK fit including both $\gamma$ and $\delta$. (e) Same as (d) except with the individual contributions from $\gamma$ and $\delta$ shown separately (dotted lines). Note that $\delta$ has $\phi_{B} \simeq \pi$ (topo.). (f) Same as (e) except that $\phi_{B}$ has been set to 0 (trivial). The best fit is achieved in (e) indicating the topological nature of $\mathrm{CaAgAs}$. frequency branch [21], and are summarized in Table I. To address the topological character of $\delta$, we directly fit the oscillatory torque signal in the high-angle regime [over the field range $18-35 \mathrm{~T}$ as shown in Fig. 2(e) to minimize the spinsplitting effect] with a simplified two-component LK formula. This approach is preferred over a Landau fan analysis [Fig. 3(b)] because of the multiple frequencies involved (see SM [25] for details of the fitting procedure). Using nonlinear least squares, we used the torque fitting formula $\tau_{\mathrm{osc}}(B)$ to directly extract the phases of the two orbits close to $\theta=90^{\circ}$, as shown in Fig. 3(a). Torque is a signed quantity, but in our measurements we are only certain of the magnitude of the signal. Assuming that the sign of torque is negative (i.e., $\mathrm{CaAgAs}$ is diamagnetic), we find from direct fitting that the oscillatory torque data close to $\theta=90^{\circ}$ is best described when the Berry phase of $\gamma$ is $\phi_{B}=0$ and that of $\delta$ is $\phi_{B}=\pi$, as shown in Figs. 3(c)-3(f). This finding provides strong evidence for nontrivial band topology in CaAgAs. Furthermore, as the angle is reduced from $\theta=90^{\circ}, \gamma$ accumulates additional Berry phase [Fig. 3(a)]. This is consistent with the lack of inversion symmetry of CaAgAs, which allows Berry phases to vary away from high-symmetry directions.

Conclusions. In this work, we have used torque magnetometry to investigate the geometry and topology of the Fermi surface of the proposed TNLSM CaAgAs. We analyze the experimentally observed quantum oscillations using theoretical input from both $a b$ initio simulations and semiclassical analysis of a simplified model. This allows us to link the observed oscillation frequencies to the coexistence of multiple cyclotron orbits with both nontopological and topological Berry phases. This provides strong evidence that the Fermi surface of hole-doped $\mathrm{CaAgAs}$ originates from an underlying topological line node at perfect stoichiometry (possibly weakly gapped by small SOC). We find no sign of any other bands near the Fermi energy, making CaAgAs an ideal setting to study TNLSMs and proximate insulating and semimetallic phases. Similar studies of samples with different doping levels may be able to reveal finer details of the nontrivial Fermi surface, and probe other fascinating phenomena enabled by the interplay of symmetry and band topology in $\mathrm{CaAgAs}$ and related materials.

Note added in proofs. Another torque study of CaAgAs up to $18 \mathrm{~T}$ Ref. [28] suggests that spin-splitting effects would also affect the phase of quantum oscillations. This study identifies a similar Fermi surface of $\mathrm{CaAgAs}$ to that reported here.

In accordance with the EPSRC policy framework on research data, access to the data will be made available [29].

Acknowledgments. We thank Ni Ni for provision of related crystals that were not part of this study. This work was mainly supported by EPSRC (EP/I004475/1, EP/I017836/1). A.I.C. acknowledges support from an EPSRC Career Acceleration Fellowship (EP/I004475/1). S.A.P. acknowledges support from the European Research Council (ERC) under the European Union Horizon 2020 Research and Innovation Programme (Grant Agreement No. 804213-TMCS). Y.H.K. acknowledges support from NSF Grant No. DMR-1455366 during the early stages of this project. A portion of this work 
was performed at the National High Magnetic Field Laboratory, which is supported by National Science Foundation Cooperative Agreement No. DMR-1157490 and the State of Florida. Part of this work was supported by HFML-RU/FOM and LNCMI-CNRS, members of the European Magnetic Field Laboratory (EMFL), and by EPSRC (UK) via its membership to the EMFL (Grant No. EP/N01085X/1). We also acknowledge the Oxford Centre for Applied Superconductivity.
[1] A. A. Burkov, M. D. Hook, and L. Balents, Phys. Rev. B 84, 235126 (2011).

[2] C. Fang, Y. Chen, H.-Y. Kee, and L. Fu, Phys. Rev. B 92, 081201(R) (2015).

[3] C. Fang, H. Weng, X. Dai, and Z. Fang, Chin. Phys. B 25, 117106 (2016).

[4] C.-K. Chiu and A. P. Schnyder, Phys. Rev. B 90, 205136 (2014).

[5] Y. Kim, B. J. Wieder, C. L. Kane, and A. M. Rappe, Phys. Rev. Lett. 115, 036806 (2015).

[6] R. Yu, H. Weng, Z. Fang, X. Dai, and X. Hu, Phys. Rev. Lett. 115, 036807 (2015).

[7] G. Bian, T.-R. Chang, R. Sankar, S.-Y. Xu, H. Zheng, T. Neupert, C.-K. Chiu, S.-M. Huang, G. Chang, I. Belopolski et al., Nat. Commun. 7, 10556 (2016).

[8] L. M. Schoop, M. N. Ali, C. Straßer, A. Topp, A. Varykhalov, D. Marchenko, V. Duppel, S. S. Parkin, B. V. Lotsch, and C. R. Ast, Nat. Commun. 7, 11696 (2016).

[9] M. Neupane, I. Belopolski, M. M. Hosen, D. S. Sanchez, R. Sankar, M. Szlawska, S.-Y. Xu, K. Dimitri, N. Dhakal, P. Maldonado, P. M. Oppeneer, D. Kaczorowski, F. Chou, M. Z. Hasan, and T. Durakiewicz, Phys. Rev. B 93, 201104(R) (2016).

[10] G. Bian, T.-R. Chang, H. Zheng, S. Velury, S.-Y. Xu, T. Neupert, C.-K. Chiu, S.-M. Huang, D. S. Sanchez, I. Belopolski, N. Alidoust, P.-J. Chen, G. Chang, A. Bansil, H.-T. Jeng, H. Lin, and M. Z. Hasan, Phys. Rev. B 93, 121113(R) (2016).

[11] Y.-H. Chan, C.-K. Chiu, M. Y. Chou, and A. P. Schnyder, Phys. Rev. B 93, 205132 (2016).

[12] S. T. Ramamurthy and T. L. Hughes, Phys. Rev. B 95, 075138 (2017).

[13] Y. Huh, E.-G. Moon, and Y. B. Kim, Phys. Rev. B 93, 035138 (2016).

[14] A. Yamakage, Y. Yamakawa, Y. Tanaka, and Y. Okamoto, J. Phys. Soc. Jpn. 85, 013708 (2016).

[15] X.-B. Wang, X.-M. Ma, E. Emmanouilidou, B. Shen, C.-H. Hsu, C.-S. Zhou, Y. Zuo, R.-R. Song, S.-Y. Xu, G. Wang,
L. Huang, N. Ni, and C. Liu, Phys. Rev. B 96, 161112(R) (2017).

[16] E. Emmanouilidou, B. Shen, X. Deng, T.-R. Chang, A. Shi, G. Kotliar, S.-Y. Xu, and N. Ni, Phys. Rev. B 95, 245113 (2017).

[17] Y. Okamoto, T. Inohara, A. Yamakage, Y. Yamakawa, and K. Takenaka, J. Phys. Soc. Jpn. 85, 123701 (2016).

[18] J. Nayak, N. Kumar, S.-C. Wu, C. Shekhar, J. Fink, E. D. L. Rienks, G. H. Fecher, Y. Sun, and C. Felser, J. Phys.: Condens. Matter 30, 045501 (2018).

[19] D. Takane, K. Nakayama, S. Souma, T. Wada, Y. Okamoto, K. Takenaka, Y. Yamakawa, A. Yamakage, T. Mitsuhashi, K. Horiba et al., npj Quantum Mater. 3, 1 (2018).

[20] A. Mewis, Z. Naturforsch. B: J. Chem. Sci. 34, 14 (1979).

[21] C. Li, C. M. Wang, B. Wan, X. Wan, H.-Z. Lu, and X. C. Xie, Phys. Rev. Lett. 120, 146602 (2018).

[22] L. Oroszlány, B. Dóra, J. Cserti, and A. Cortijo, Phys. Rev. B 97, 205107 (2018).

[23] H. Yang, R. Moessner, and L.-K. Lim, Phys. Rev. B 97, 165118 (2018).

[24] D. Shoenberg, Magnetic Oscillations in Metals, Cambridge Monographs on Physics (Cambridge University Press, Cambridge, UK, 1984).

[25] See Supplemental Material at http://link.aps.org/supplemental/ 10.1103/PhysRevResearch.2.012055 for data analysis and theoretical analysis details, and additional figures.

[26] A. Alexandradinata and L. Glazman, Phys. Rev. Lett. 119, 256601 (2017).

[27] J. Wang, J. Niu, B. Yan, X. Li, R. Bi, Y. Yao, D. Yu, and X. Wu, Proc. Natl. Acad. Sci. USA 115, 9145 (2018).

[28] H. T. Hirose, T. Terashima, T. Wada, Y. Matsushita, Y. Okamoto, K. Takenaka, and S. Uji, Real spin and pseudospin topologies in the noncentrosymmetric topological nodal-line semimetal CaAgAs, arXiv:2002.05597 [cond-mat.str-el].

[29] A. Coldea, P. Reiss, and Y. Kwan, Quantum oscillations probe the Fermi surface topology of the nodal-line semimetal CaAgAs, University of Oxford, 2020, doi:10.5287/bodleian:v0BM5PR4P. 